\title{
Predicting mechanical degradation indicators of silver fir wooden strips using near infrared spectroscopy
}

\author{
Jean Baptiste Barré ${ }^{1,2}$ - Franck Bourrier ${ }^{1,2}$ - Lauric Cécillon ${ }^{1,2}$ • Loïc Brancheriau ${ }^{4}$. \\ David Bertrand $^{3} \cdot$ Marie France Thévenon ${ }^{4}$ Freddy Rey ${ }^{1,2}$
}

\begin{abstract}
The management of ecological engineering structures making up a timber structure requires periodical evaluations, including the level of decay of the constituent parts of the timber structure. Methods exist to measure the level of decay in the laboratory or in the field. However, they are rarely suitable for the conditions of ecological engineering structures, or give partial information. The aim of this study was to predict two mechanical degradation indicators $\left(D w_{M O E}\right.$ and $\left.D w_{M O R}\right)$ of silver fir (Abies alba) wooden strips during microbial decomposition using nearinfrared spectroscopy (NIRS). For 1.5 years, the degradation of 180 squared wooden strips, $30 \mathrm{~mm}$ wide and 500 $\mathrm{mm}$ long, buried in a greenhouse near Grenoble, France (Altitude: $200 \mathrm{~m}$ ) was monitored. $D w_{M O E}$ and $D w_{M O R}$ were set from the normalized losses in modulus of elasticity (MOE) and modulus of rupture (MOR), two mechanical properties classically used for timber-structure design. A calibration set of 109 samples was selected to build two separate predictive models of $D w_{M O E}$ and $D w_{M O R}$ using partial least square regression. The NIR-based models applied to a validation set of 47 samples indicated good prediction performance. The model has a root mean square error of prediction (RMSEP) of 0.15 and a coefficient
\end{abstract}

Jean Baptiste Barré

jean-baptiste.barre@irstea.fr

1 IRSTEA, 2, Rue de la Papeterie, 38402 Saint-Martin-d'Hères, France

2 Université Grenoble Alpes, 38402 Grenoble, France

3 INSA-Lyon (National Institute of Applied Sciences of Lyon), 20, Avenue Albert Einstein, 69621 Villeurbanne Cedex, France

4 CIRAD, UR BioWooEB UMR AMAP, 34398 Montpellier Cedex 5, France of determination $\left(r_{p}^{2}\right)$ of 0.79 . The $D w_{M O R}$ model has a RMSEP of 0.13 and a $r^{2}$-value of 0.91 . These results highlight the considerable potential of NIRS in assessing the extension of decay in wooden logs.

\section{Introduction}

Ecological engineering structures, such as wooden check dams and timber log crib walls, are structurally based on timber structures. These structures, made from a framework of logs, require specific attention since wood may be degraded by a microbial community composed of a variety of micro-organisms or insects. In wet conditions, the most efficient ones are the wood-rotting Basidiomycetes (brown and white rots), Ascomycetes (soft and white rots) and Deuteromycetes (soft rot) that use the wood?s chemical components for their nutrition. This results in losses in wood strength and stiffness with potential risk for the structure's integrity. For that reason, practitioners have based their field investigations mainly on direct or indirect density measurement such as resistance drilling or penetration methods (Kasal and Tannert 2011; Mkip and Linkosalo 2011) and visual inspections. However, wood has already lost significant strength when fungi effects become visible and the mass loss is quantifiable (Wilcox 1978). Alternative approaches used, for example, on time-domain reflectometry water content (Previati et al. 2012) and stress-wave measurement (Dackermann et al. 2013) provide experts with more relevant information. These approaches are based on the in-situ measurement of wood decay indicators such as mass loss, hardness loss and mechanical property variations.

Specific gravity is a widely used indicator to quantify the extent of fungal attack. However, strength (Haines et al. 
1996) and stiffness (Przewloka et al. 2008) losses are more suitable indicators at the early stage of decay (Curling et al. 2002). The fungi actually alter the lignocellulosic matrix by various mechanisms (Blanchette 1995), and therefore the mechanical properties (Rowell 2012). Mechanical properties are all the more important since structural design according to the Eurocode 5 standard (CEN 2005) is based on these properties. Thus, the modulus of elasticity (MOE) and the modulus of rupture (MOR) are of interest both for structural design and to define decay indicators. However, MOE and MOR remain impossible to measure in-situ using classical methods because a bench test is necessary. An alternative solution lies in the use of the near infrared spectroscopy (NIRS) applied to milled cores sampled on structure parts to predict changes in MOE and MOR.

Functional groups of the wood molecules submitted to an electromagnetic radiation vibrate and absorb a part of the radiation. NIRS (wavelengths from 800 to $2500 \mathrm{~nm}$ ) measures this absorbance or reflection depending on the device used. The returned spectrum is analysed statistically to extract the meaningful information. The spectral data are often used to build predictive models of variables characterizing the data set. Previous studies (Tsuchikawa and Schwanninger 2013) have shown that MOE and MOR could be predicted from near infrared (NIR) spectra using multivariate statistics. Kelley et al. (2004) obtained not only accurate predictions of these mechanical properties for solid loblolly pine (Pinus taeda) samples, but also, of their chemical compounds, i.e. lignin, glucose, mannose and extractives. Sandak et al. (2015a) also reported that NIRS has been used to investigate degradation in wood structural members. Fackler et al. (2007c) analysed chemical changes due to the fungal activity. Green et al. (2010b) predicted levels of degradation in southern pine (Pinus spp.). Kelley et al. (2002) detected the chemical changes associated with brown-rot biodegradation of spruce wood.

However, they inoculated samples with only one fungus at a time and stored them in an incubator controlling humidity and temperature. On the contrary, wood inoculated with a microbial community has never been studied by NIRS to assess the level of decay.

The aim of this study was to assess the decay extent of silver fir wooden strips degraded by a microbial community using NIRS in semi-controlled conditions. First, the level of decay was defined from two degradation indicators $D w_{M O E}$ and $D w_{M O R}$. They were set from the normalized losses in MOE and in MOR, respectively, between intact and decayed states. Then, partial least square regressions were performed between spectra and $D w_{M O E}$ or $D w_{M O R}$ to establish a predictive model of these indicators.

\section{Materials and methods}

\subsection{Wood samples preparation}

As intra-tree mechanical properties variation is greater than inter-tree variation (Gonzalez-Rodrigo et al. 2013), a single freshly-cut log of European silver fir (Abies alba sp.) was selected to supply the 209 samples $\left(600 \times 30 \times 30 \mathrm{~mm}^{3}\right)$. No specific attention was paid to defects such as knots. Two pieces sized $50 \times 30 \times 30 \mathrm{~mm}^{3}$ were collected from each sample: one for moisture content (MC) measurement and the other for NIRS acquisition. The residual pieces sized $500 \times 30 \times 30 \mathrm{~mm}^{3}$ were used for mechanical tests (intact state), then for the degradation process.

The degradation process took place inside a greenhouse between March, 2013 and October, 2014 in Grenoble, France (Fig. 1a). The progression of decay was assumed to be heterogeneous in the cross-section and in the longitudinal direction. Nevertheless, the samples have been buried to limit the influence of heterogeneous fungal growth in the longitudinal direction compared to stakes in the field test according to the EN 252 standard (CEN 2014). One hundred and eighty of the 209 samples were buried with two layers of different materials. The first layer was made of decayed silver fir debris collected in a silver fir forest stand. This layer included an appropriate fungal community that
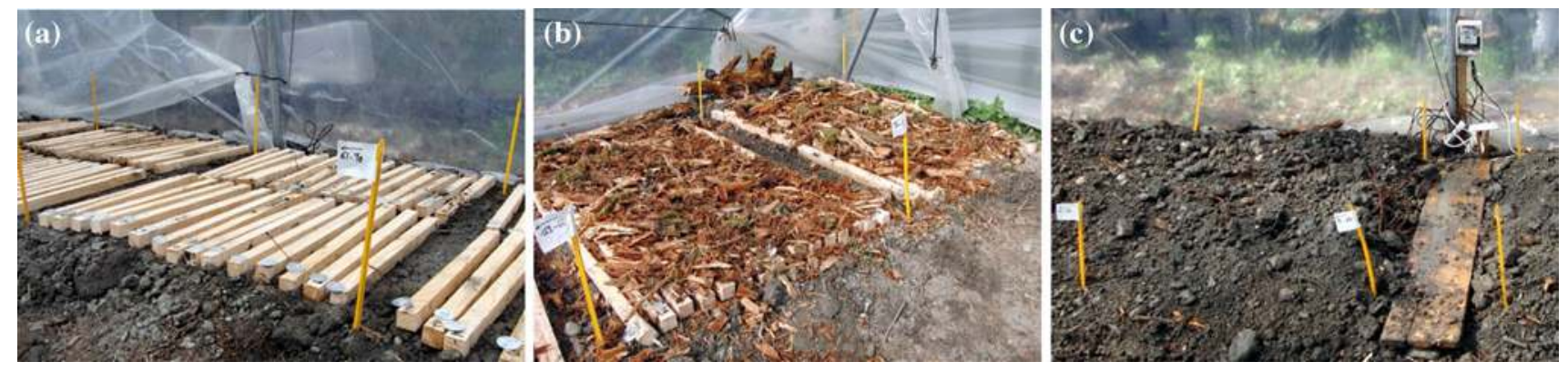

Fig. 1 Successive steps of sample installation in the greenhouse. a Intact samples divided into series; $\mathbf{b}$ samples covered with the first layer of woody debris; $\mathbf{c}$ samples covered by the second layer of soil 
had already been active (Fig. 1b). The second is a $5-\mathrm{cm}$ local soil layer (Fig. 1c). The only controlled parameter during the degradation process was the $\mathrm{MC}$ of the samples, which was kept by soil humidification above the fibre saturation point (FSP), i.e. $29 \%$ according to the Tropix database (CIRAD 2011). The resistive wood moisture sensor Humitest Timb plus (Domosystem) was used to check MC in-situ. The 29 remaining samples were used to be mechanically tested until rupture at the intact state.

In the greenhouse, the samples were divided into six sets of 30 samples. The sets were successively removed from the greenhouse after 44, 73, 112, 149, 178 and 505 days. The decayed samples were prepared for measurements similar to that for the intact state: for each sample, two pieces sized $50 \times 30 \times 30 \mathrm{~mm}^{3}$ were collected for NIRS and density measurement. The residual sample,sized $400 \times 30 \times 30$ $\mathrm{mm}^{3}$, was kept for the mechanical test. Each sample was cleaned of soil and mycelium by careful brushing prior to measurement.

\subsection{Measurement of moisture content and mechanical properties}

Measurement of the MC and mechanical properties was based on the assumption that they were equally distributed within the entire specimen. The MC of the samples was calculated for both intact and decayed pieces (Eq. 1). The oven-dry weight $\left(W_{0}\right)$ was measured after sample drying until weight stabilization using a Memmert oven set at $103.5^{\circ} \mathrm{C}$.

$M C=\left[\left(W_{h}-W_{0}\right) / W_{0}\right] \times 100$

where $W_{h}$ is the wet weight.

MOE and MOR were measured using three point bending tests shortly after taking the samples from the greenhouse so that the variation in MC could be ignored. The cross-head loading velocity for all measurements was 2 $\mathrm{mm} / \mathrm{min}$ to remain in quasi-static conditions. The MOE was calculated in the initial linear part of the load-deflection curve using Eq. 2. For practical reasons, the span-to-depth ratio was limited to 13.33 for intact samples and 10.67 for decayed samples. As a consequence, the measured deflection $f$ included both bending and shear effects. In this case, the calculated MOE corresponded to an apparent modulus of elasticity.

$M O E=\frac{F \cdot L^{3}}{48 \cdot f \cdot I_{G}}$

where $F$ is the applied force, $L$ the length between the two supports, $f$ the deflection of the sample corrected by the indentations at the supports and the head-load, and $I_{G}$ the moment of inertia of the cross section.
The MOR is defined from the maximum load supported by the sample using Eq. 3 (Dinwoodie 2000b).

$M O R=\frac{3 \cdot F_{\max } \cdot l}{2 \cdot b \cdot h^{2}}$

where $F_{\max }$ is the maximum applied force, 1 the length between the two supports, $b$ the sample width, $h$ the sample height.

From these two properties, two mechanical indicators $D w_{M O E}$ and $D w_{M O R}$ were defined depending on each sample's rate of degradation. They are considered as reference values used to build the predictive models. $D w_{M O E}$ is the normalized loss in the MOE of the same sample between intact and decayed states (Eq. 4).

$D w_{M O E}=\frac{M O E_{i}-M O E_{d}}{M O E_{i}}$

where $M O E_{i}$ and $M O E_{d}$ are the MOE of a sample in intact and decayed states, respectively.

$D w_{M O R}$ is the normalized loss in the MOR. The MOR cannot be evaluated in intact and decayed states for the same sample because it has to be brought to failure to measure the MOR. Thus, the MOR of a set of 29 samples, randomly selected amongst 209 and not buried in the greenhouse were measured and averaged into one $M O R^{*}{ }_{i}$ value. $D w_{M O R}$ was next calculated for each sample according to Eq. 5.

$D w_{M O R}=\frac{M O R_{i}^{*}-M O R_{d}}{M O R_{i}^{*}}$

where $M O R^{*}{ }_{i}$ is the mean MOR of 29 samples in the intact states, $M O R_{d}$ the MOR of the sample in the decayed state.

This definition of the indicators based on normalization allows one to limit the shear effect assuming that it remains the same, whatever the level of decay. Furthermore, given that the samples's MC was kept above FSP by watering, it was assumed that MC differences had no influence on the mechanical properties and consequently on the indicators (Dinwoodie 2000a).

\subsection{Near infrared spectroscopy measurements}

A piece of each intact or decayed sample, a transversal slice measuring $50 \times 30 \times 30 \mathrm{~mm}^{3}$, was collected for the NIRS measurement and dried for $12 \mathrm{~h}$ in an oven set at $40{ }^{\circ} \mathrm{C}$ to ease the grinding. In the next step, these cut off parts of the samples were milled to a fine powder $(0$, $25 \mathrm{~mm}$ ) in a Retsch ZM 200 mill. They were ground to reduce the spatial heterogeneity of wood properties existing in intact wood and reinforced by the fungal colonization (Schwarze 2007). The powder was dehydrated again 
for $12 \mathrm{~h}$ in an oven to limit the adverse effect of water during spectrum acquisition. Intact NIRS-sample refers to the powder obtained from an intact sample and intact spectrum to the corresponding infrared spectrum. Likewise, the powder obtained from the decayed sample was called decayed NIRS-sample and its spectrum decayed spectrum. The NIRS measurements were taken using a diffuse reflectance module with the Thermo Scientific Antaris 2 FT-NIR analyser. This instrument records the absorbance in the spectral region $\left(3999,10,001 \mathrm{~cm}^{-1}\right)$ at a resolution of $4 \mathrm{~cm}^{-1}$. The spectra are thus composed of 1557 wavenumbers. With the same parameters, spectra were acquired from cellulose and lignin kraft extracted from Pinus pinaster sp. samples to ease spectrum interpretations.

\subsection{Spectral data treatments}

The information contained in the spectra were highlighted by specific treatments. All data processing was done using $\mathrm{R}$ software (R Core Team 2013). The spectra were managed using the hyperSpec package (Beleites and Sergo 2012). The pre-processing step followed recommendations of Rinnan et al. (2009) for limiting the scattering effect usually observed with milled samples and adjusting the baseline shifts between samples. A single pre-processing step was performed on the spectra to limit the model's complexity. The spectra were adjusted by a de-trending method (baseline correction) using the baseline package (Liland and Mevik 2014).

The proposed method aims to assess the level of decay by comparing of intact and decayed spectra. A differential spectrum $S_{\text {diff }}$ was calculated for each decayed sample from the difference between a mean intact spectrum, the average of all intact spectra, and the spectrum of a decayed NIRSsample (Eq. 6). This methodology was selected for two reasons. Firstly, it was considered that intact spectra would not be available throughout real structure investigations. The proposal aims to consider the mean intact spectrum to establish a spectral reference for the silver fir samples. Secondly, the saproxylic community present in the greenhouse was potentially highly diverse (Stokland et al. 2012) and was not determined for practical reasons. In particular, the three types of wood-inhabiting fungus (brown, white and soft rots) might be expected. No assumption can be made on a predominantly decayed chemical compound. However, no studies have been published on the effect of the fungal community on NIR spectra. Most NIRS studies of decayed wood have investigated spectral modifications caused by known fungal species in controlled conditions. These species are mainly white or brown rot types (Fackler and Schwanninger 2012; Green et al. 2012) and seldom soft rot (Stirling et al. 2007). Hence, it was proposed to highlight the affected parts of the spectra by means of $S_{d i f f}$. $a b s_{\text {diff }}^{i}=a b s_{\text {g.mean }}^{i}-a b s_{d}^{i}$

where $i$ indicates the wavelength, $a b s_{\text {g.mean }}^{i}$ is the baselinecorrected absorbance of the mean intact spectrum and $a b s_{d}^{i}$ is the baseline-corrected absorbance of the decayed sample.

\subsection{Spectral analysis}

The data set exploration started with a principal component analysis (PCA) on the whole spectra. PCA facilitates the understanding of complex data sets with many variables. The partial least square regression (PLSR) was next used to develop predictive models of $D w_{M O E}$ and $D w_{M O R}$ from differential spectra. PLSR is a multivariate regression method used in spectroscopy because it is adapted when collinear variables are used (Wold et al. 2001). Sandak et al. (2015b) referred to several studies using PLSR in a quantitative manner in decayed-wood investigations. The process of model construction was divided into calibration and validation phases (Fig. 2).

The validation phase consists of applying the PLSR function from the pls package (Mevik et al. 2013) to a calibration set of 109 samples using the Kennard-Stone algorithm, as implemented in the soil.spec package (Sila and Terhoeven-Urselmans 2013). In this phase, the models were internally validated by the leave-oneout (LOO) cross-validation method. The quality of the PLSR models was assessed using the coefficient of multiple determination $r_{c}^{2}$ and the root mean squared error of calibration (RMSEC), i.e. the residuals of the calibration data. Likewise, the results of the cross validation has been characterized by $r_{c v}^{2}$, the root mean squared error of cross-validation (RMSECV) (Mevik and Cederkvist 2004). The validation phase corresponded to outer tests with a validation data set of 47 samples separated from the calibration set. It consists of applying the model calibrated in the previous phase to the validation set to predict the two indicators $D w_{M O E}$ and $D w_{M O R}$. The predictive ability of the model is evaluated by the coefficient of determination $r_{p}^{2}$, the root mean squared error of

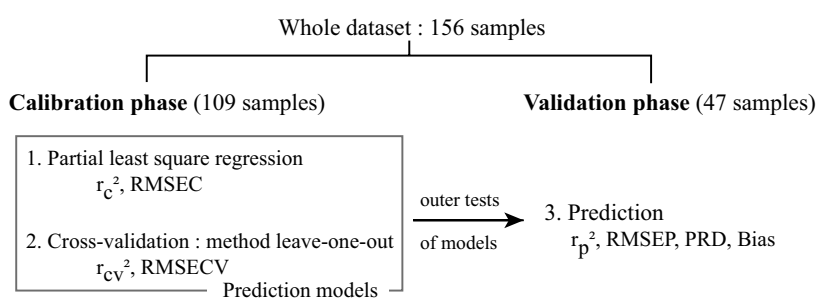

Fig. 2 Details of the validation process of the predictive models 
prediction (RMSEP), the ratio of performance to deviation (RPD), calculated as the ratio of the standard deviation of the reference data to the RMSEP (Schimleck et al. 2003), and the bias, calculated as the difference of the mean of the predicted values versus the mean of the true values (Bellon-Maurel et al. 2010). The value of the bias should be close to 0 for accurate calibration.

\subsection{Wavelength selection}

Following the recommendations of Schwanninger et al. (2011), the spectral range was reduced to improve the model's performance. The principle adopted consisted in retaining regions where differences between decay and the mean intact spectra were considered high. For this purpose, a process in two-step was developed. Firstly, the standard deviation (SD) of absorbances $a b s_{\text {diff }}^{i}$ among the different $S_{\text {diff }}$ spectra was calculated for each wavelength. Only the wavelengths corresponding to SD larger than a threshold value $S D_{\text {lim }}$ were kept in the PLSR. Secondly, an iterative test was developed to determine the best wavelength range, i.e. those that minimize RMSEP. Thus, different thresholds were tested by varying $S D_{\text {lim }}$ from 0 to the optimal $S D_{\text {lim }}$ which corresponded to the lower RMSEP. Furthermore, the laboratory climate was not fully controlled and dried wood samples are very hydrophilic. Thus, the wavelengths associated with water (5150-5220, 5051, $7073 \mathrm{~cm}^{-1}$ ) were removed from the analysis, even if they corresponded to wavelengths associated with OH-bonds of wood compounds (Schwanninger et al. 2011). OH-bonds are nevertheless related to other wavelengths, in particular those from 4620 to $4890 \mathrm{~cm}^{-1}$, where spectra variations due to fungi were observed (Sandak et al. 2013).

\section{Results and discussion}

The aim of this study was to quantify the level of decay of silver fir strips degraded by a fungal community using NIRS. For this purpose, reference indicators $D w_{M O E}$ and $D w_{M O R}$ were defined from MOE and MOR measured in the intact and decayed states. The intact wooden strips were buried in a greenhouse for a period of 44-505 days. After the decay process, the evidence of fungal activity was visually observed at the surface of the samples. Mycelium was noted on all samples with variable intensities in terms of spatial distribution (Fig. 3). Small cracks were present on the most severely decayed samples. The slicing of samples made it possible to visually ascertain that discolourations were present inside the wood to a variable extent. It was assumed that fungi penetrated wood a short time after inoculation.

\subsection{Mechanical degradation indicators $D w_{M O E}$ and $D w_{M O R}$}

For the 109 samples of the calibration set, the mean MOE was $8211 \mathrm{MPa}$ (i.e $\mathrm{N} / \mathrm{mm}^{2}$ ) (SD $951 \mathrm{MPa}$; Table 1). The mean MOR, calculated from 29 of the intact samples, was 41.9 MPa (SD 7.0 MPa). The mean MC was $106 \%$, always greater than $30 \%$. For the 47 samples of the validation set, the mean MOE was $8172 \mathrm{MPa}$ (SD $829 \mathrm{MPa}$ ). The mean MC was $113 \%$. For decayed samples, the mean MOE was $3889 \mathrm{MPa}$ and $2966 \mathrm{MPa}$ (SD 2191 and $2486 \mathrm{MPa}$ ) for the calibration and validation sets, respectively. The mean MOR was 30.1 and 23.9 MPa (SD 14.2 and 17.1 MPa) for the calibration and validation sets, respectively. For the last series (i.e. 505 degradation days, 25 samples), the MOE and MOR were set at $0 \mathrm{MPa}$ because the samples were too degraded to be tested using bending tests and could easily be broken by hand. The MOE and MOR were correlated in the intact and decayed states (Fig. 4). Indeed, an increase
Fig. 3 a Mycelium observed on the surface of a sample degraded for 73 days. The tape measure is in centimetre. $\mathbf{b}$ The mycelium may have a higly variable spatial extent. Sample degraded for 112 days
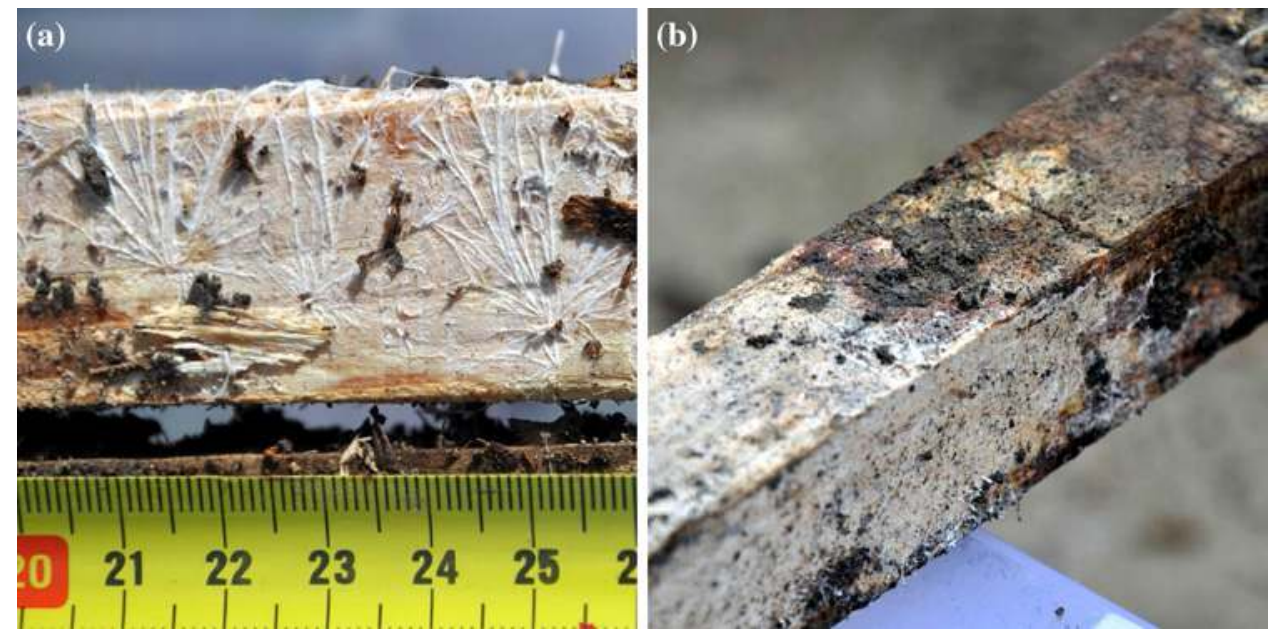
Table 1 Physical and mechanical measurements and indicators of the level of decay

\begin{tabular}{|c|c|c|c|c|c|c|c|c|}
\hline \multirow[b]{2}{*}{ Wood property } & \multicolumn{4}{|c|}{ Calibration set, $\mathrm{n}=109$} & \multicolumn{4}{|c|}{ Validation set, $n=47$} \\
\hline & Mean & Min. & Max. & SD & Mean & Min. & Max. & SD \\
\hline \multicolumn{9}{|l|}{ Intact samples } \\
\hline Moisture content (\%) & 106 & 32 & 179 & 44 & 113 & 35 & 181 & 47 \\
\hline$M O E_{\text {intact }}(\mathrm{MPa})$ & 8211 & 5672 & 10275 & 951 & 8172 & 5792 & 9460 & 829 \\
\hline$M O R_{\text {intact }}(\mathrm{MPa})^{\mathrm{a}}$ & 41.9 & 28.3 & 56.6 & 7.0 & - & - & - & - \\
\hline \multicolumn{9}{|l|}{ Decayed samples } \\
\hline Moisture content (\%) & 104 & 42 & 175 & 37 & 106 & 47 & 170 & 35 \\
\hline$M O E_{\text {decay }}(\mathrm{MPa})$ & 3889 & 0 & 9001 & 2191 & 2966 & 0 & 6905 & 2486 \\
\hline$M O R_{\text {decay }}(\mathrm{MPa})$ & 29.8 & 0 & 53.3 & 14.2 & 23.9 & 0 & 49.9 & 17.1 \\
\hline \multicolumn{9}{|l|}{ Indicators } \\
\hline$D w_{M O E}(-)$ & 0.52 & 0.06 & 1 & 0.27 & 0.64 & 0.13 & 1 & 0.30 \\
\hline$D w_{M O R}(-)$ & 0.29 & -0.27 & 1 & 0.34 & 0.43 & -0.19 & 1 & 0.42 \\
\hline
\end{tabular}

Range and standard deviation (SD)

${ }^{a}$ Measured for 29 intact samples

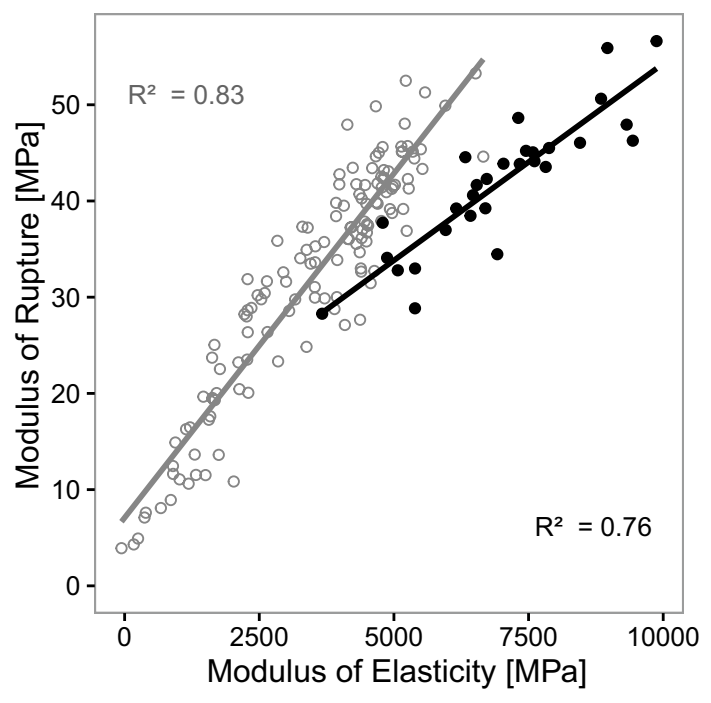

Fig. 4 Relationship between the MOE and MOR in intact (black points) and decayed (grey points) states

in MOE induces an increase in MOR. Assuming a linear relationship between these two variables gives coefficients of determination $r^{2}$ of 0.72 and 0.86 , respectively.

The MOE and MOR measurements in decayed conditions were skewed by the heterogeneity of fungal decay visually observed in the cross-section given that Eqs. 2 and 3 were defined assuming an isotropic material. However, it gave coherent values from a decay-assessment perspective. Thus, the degradation process tested in the greenhouse provided a large data set of $156 D w_{M O E}$ and $D w_{M O R}$ values (Fig. 5). Regarding $D w_{M O R}, 28$ negative values ranging from -0.27 to 0 (mean of negative $D w_{M O R}:-0.09$ ) were observed as the MOR of decayed samples was compared to a mean MOR of intact samples. This is in particular the consequence of the MOR's variability rated at $9 \mathrm{MPa}$ (SD

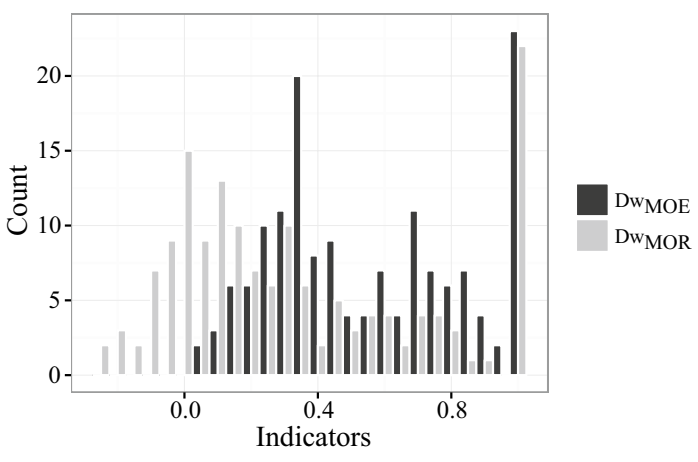

Fig. 5 Distribution of the mechanical degradation indicators $D w_{M O E}$ (dark) and $D w_{M O R}$ (grey)

for silver fir-Tropix 7). From a mechanical point of view, both indicators have different meanings and appear to be complementary in the context of timber structure design and monitoring. $D w_{M O E}$ is related to the bending stiffness and therefore linked to wooden beam deformation. $D w_{M O E}$ could therefore be used to assess a capacity of deformation before rupture. $D w_{M O R}$ is related to the bending strength and thus to the material rupture.

\subsection{Near-infrared spectra}

Spectrum exploration using PCA performed with all wavenumbers showed a separation between intact and decayed NIRS-samples (Fig. 7a). The first component (PC1) loadings (Fig. 7b) were characterized by three main peaks (around 4502, 5361 and $7577 \mathrm{~cm}^{-1}$ ) corresponding to wavenumbers where baseline-corrected absorbances are extremely low (Fig. 6). These three peaks were also observed on the second component (PC2) loadings (4995, 5331, 5390, 7553 and $7658 \mathrm{~cm}^{-1}$ ). The PC2 pointed to a lesser extent to five other wavenumbers: 4142, 4870, 


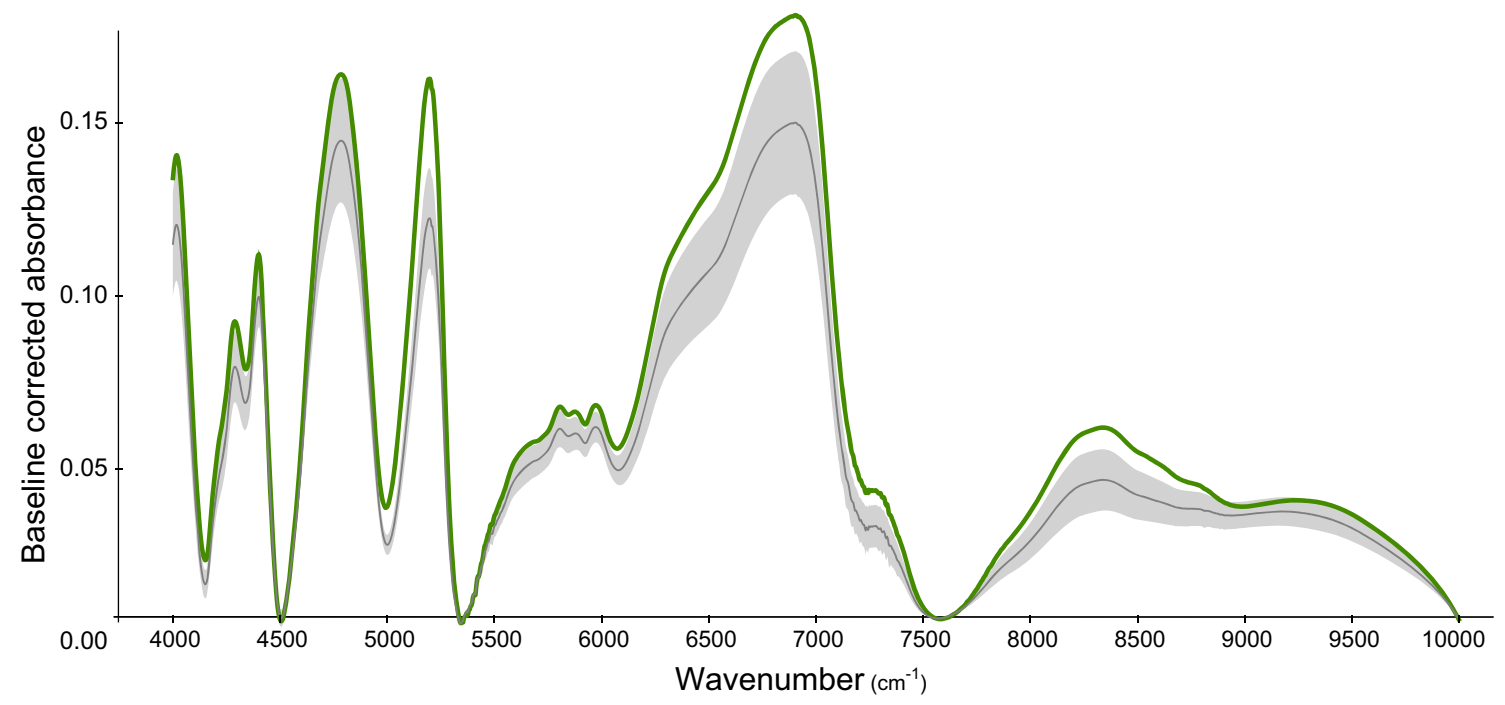

Fig. 6 Mean intact spectrum (solid line) and the mean decayed spectra (solid line with ribbon) with the ribbon that represents the absorbance standard deviation

(a)

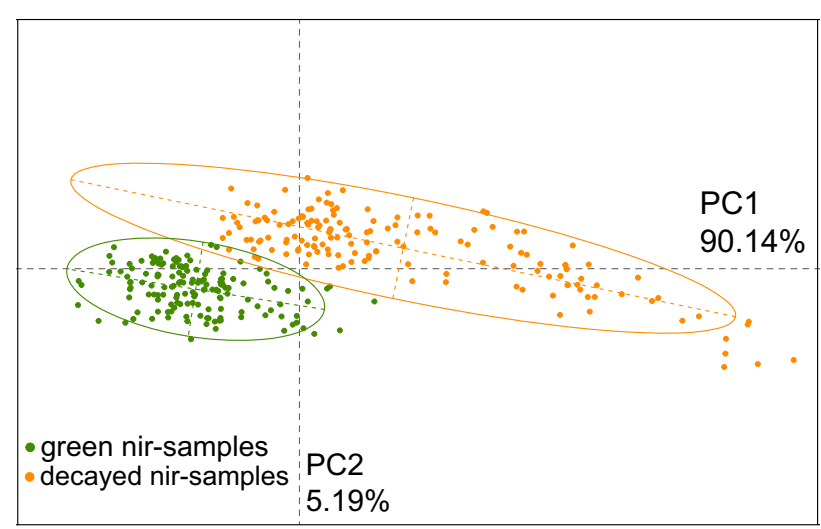

Fig. 7 a Principal component analysis of the baseline-corrected spectra of intact and decayed samples. The ellipses graphically summarize the point clouds. b Loadings of the first two principal compo-

$5022,5770,9045 \mathrm{~cm}^{-1}$. PCA performed only on decayed NIRS-samples showed a graduation of $D w_{M O E}$ (Fig. 8a) or $D w_{M O R}$ (Fig. $8 \mathrm{~b}$ ) visible along PC1 axes.

The mean intact and decayed spectra followed the same pattern but decayed spectra had lower baseline-corrected absorbance values in the whole NIR spectral range (4000-10,000 $\mathrm{cm}^{-1}$ ) (Fig. 6).

The findings of this study confirm that NIRS spectra contain relevant information regarding the microbial activity in wood, even at the early stage. The first feature highlighted by PCA (Fig. 7a) is a difference between (b)

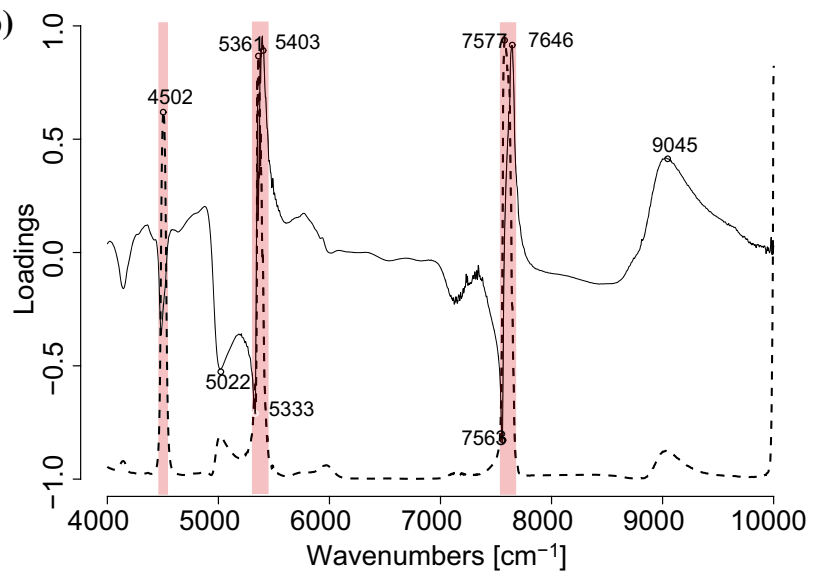

nents (dashed and solid lines, respectively). The ribbons correspond to ranges where baseline-corrected absorbance is null

the spectra of intact and decayed samples, whatever the duration of decay. This is also observed on the spectrum plot (Fig. 9). The loss in baseline-corrected absorbance of decayed spectra compared to the intact spectra is visible in regions associated with cellulose (peaks 5865 and $6727 \mathrm{~cm}^{-1}$ ) or lignin (peaks, 6875 and $5972 \mathrm{~cm}^{-1}$ ) (Schwanninger et al. 2011). Similar observations have been described with different wood species decayed by brown or white rots (Fackler et al. 2007a; Sandak et al. 2013), remembering that soft rot may be involved in the current experiment given that the samples were subjected to a microbial community. This constant difference 
Fig. 8 Principal component analysis of the baselinecorrected spectra of decayed samples. Grey scale and sizes are a function of the level of decay according to $D w_{M O E}(\mathbf{a})$ and $D w_{M O R}(\mathbf{b})$
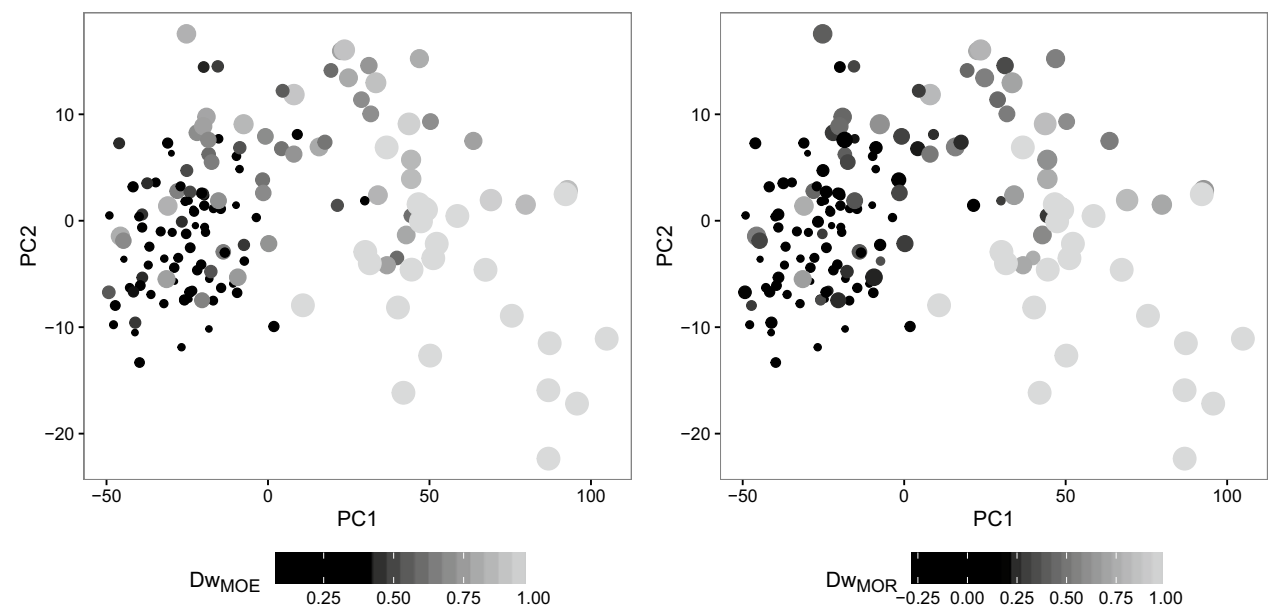

Fig. 9 Mean spectra of intact (solid line) and decayed (solid line with ribbon) silver fir samples. The ribbon corresponds to the standard deviation of decayed sample absorbance. The cellulose spectrum corresponds to the dashed line and the lignin kraft spectrum corresponds to the dotted dashed line

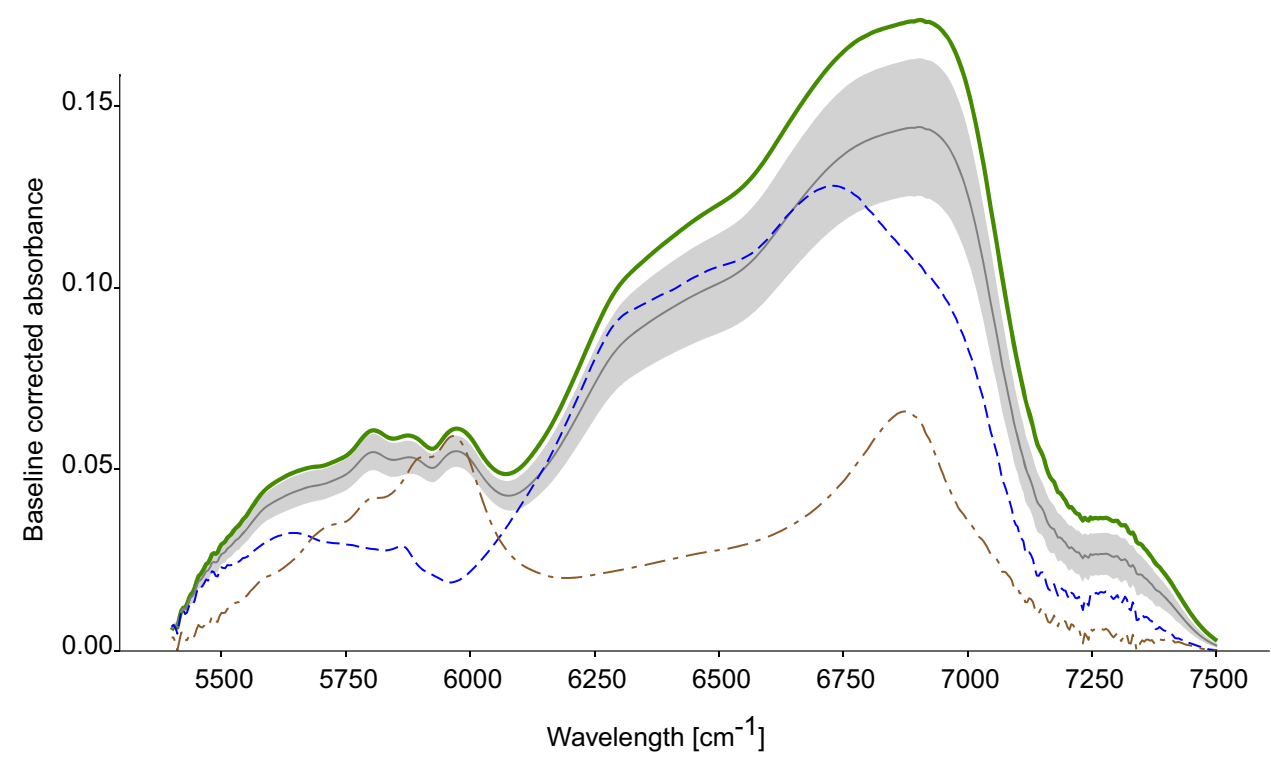

between spectra confirms the value of calculating a differential spectrum that extracts the meaningful spectral information. PCA of decayed NIRS-samples (Fig. 8) also distinguishes strongly decayed samples $\left(D w_{M O E}>0.75\right.$ and $D w_{M O R}>0.75$ ) from samples that are less decayed along the PC1 axis. PCA is, however, limited for further investigation because of an aggregation of less decayed NIRS-samples. PCA loadings (Fig. 7b) reveal no specific wavelength except one associated with $\mathrm{OH}$-bonds (peak, $5021 \mathrm{~cm}^{-1}$ ) and others where baseline-corrected absorbance is low (peaks, 4502, 5361, 7577 and $9036 \mathrm{~cm}^{-1}$ ).

The differential spectra provide evidence of some spectral regions where differences between the mean intact spectrum and decayed spectra are of interest (Fig. 11). Nevertheless, these regions are distributed all along the NIR range. PLSR performed in the whole NIR range should be sufficient to assess decay, as other studies did (Stirling et al. 2007; Leinonen et al. 2008; Green et al. 2012). But this solution would not be optimal according to suggestions by Nadler and Coifman (2005) and Cécillon et al. (2008) that prescribe wavelength range reduction to decrease the root mean square error of PLSR. This motivated the development of the wavelength selection method presented in this study.

\subsection{Prediction of mechanical degradation indicators $D w_{M O E}$ and $D w_{M O R}$ using the PLSR of differential spectra}

Wavelength selection The optimal SD values to determine wavenumbers ranges optimizing the PLSR are 0.0062 $\left(D w_{M O E}\right)$ and $0.0055\left(D w_{M O R}\right)$ (Fig. 10). Figure 11 presents the different spectral ranges kept for the PLSR. In both cases, six similar ranges were selected. Amongst the six regions identified, regions $2,3,4$ and 5 of both models (Fig. 11) had similarities to those obtained by other 

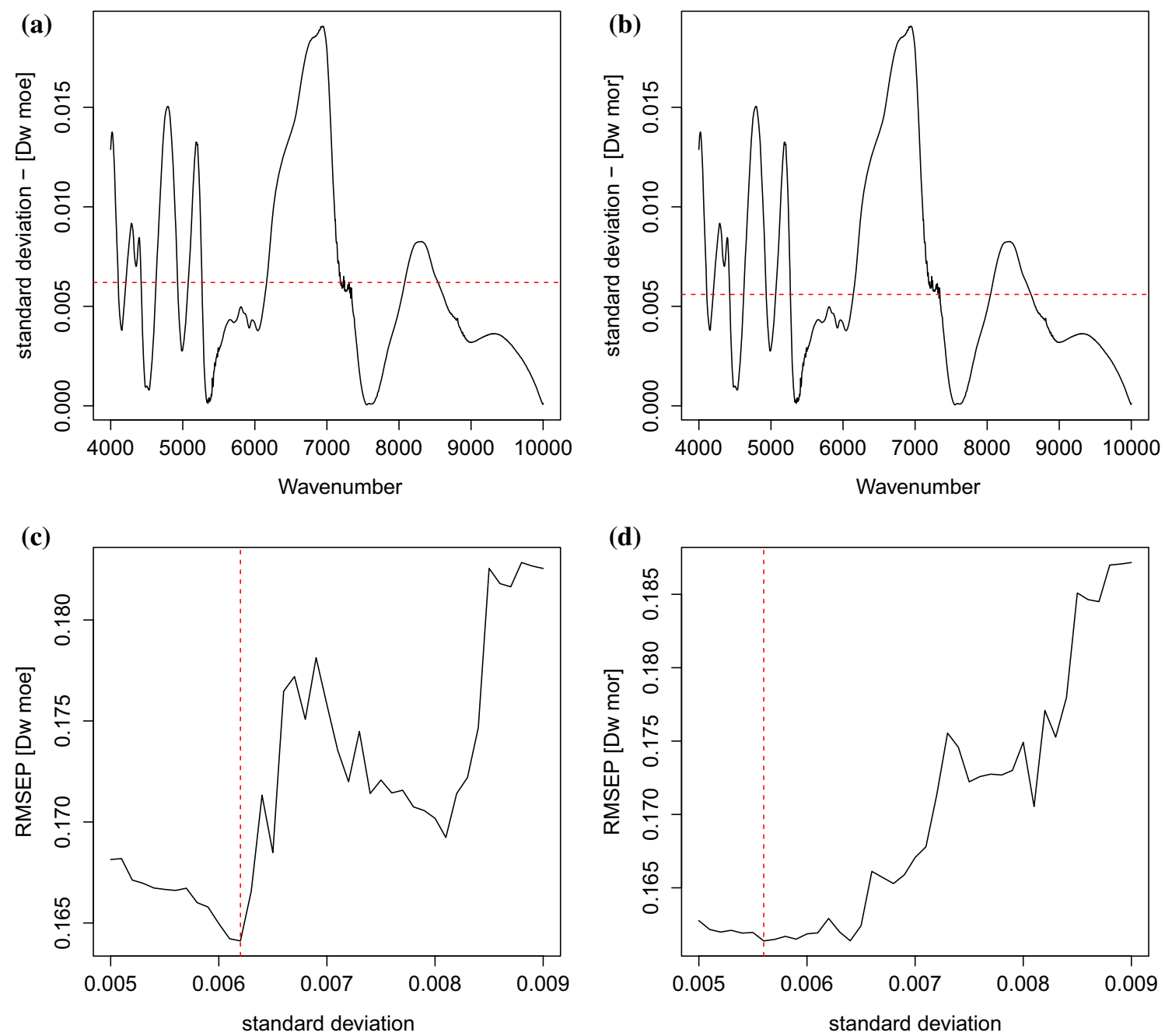

Fig. 10 Standard deviation of the differential spectra $S_{\text {diff }}$ with the optimal $S D_{\text {lim }}$ (dashed lines) for PLSR with $D w_{M O E}(\mathbf{a})$, and with $D w_{M O R}(\mathbf{b})$. RMSEP variation is a function of $S D_{\text {lim }}$ for $D w_{M O E}$ (c)

and for $D w_{M O R}(\mathbf{d})$. The dashed lines correspond to the optimal $S D_{\text {lim }}$, i.e. where RMSEP is minimal

research on decay effects on wood spectra. Fackler et al. (2007b) found the best regression for weight loss of brown rotted beech wood in the NIR region (7235-6106 and $5384-4150 \mathrm{~cm}^{-1}$ ). They reported that this model was not applicable to white rot fungi. Ishizuka et al. (2012) retained $4050-5000$ and $5800-6800 \mathrm{~cm}^{-1}$ for PLSR to predict lignin and cellulose contents. The main difference between the two studies and the results presented herein remains in the (5800-6000 $\mathrm{cm}^{-1}$ ) region associated with lignin and identified as being important in the case of white rot. This range was not included in region 5, which starts at 6163 and 6144 $\mathrm{cm}^{-1}$ for $D w_{M O E}$ and $D w_{M O R}$, respectively. It was concluded that lignin might be less affected than polysaccharides by the fungal community involved. In natural conditions, the composition of the fungal community changes over time and depends on early established and long-lived species (Ottoson 2013). Thus, further investigations of the method

on various fungal communities in natural conditions would be highly valuable.

PLSR models With these spectral ranges, two PLSR models were developed from differential spectra $S_{\text {diff }}$ to predict $D w_{M O E}$ or $D w_{M O R}$. The results obtained after the LOO validation were significant with RMSECV of 0.16 for both indicators and $r_{v}^{2}$ of 0.63 and 0.77 for $D w_{M O E}$ and $D w_{M O R}$ prediction, respectively (Table 2). These models applied to the validation set confirm the consistency of the results: to predict $D w_{M O E}$ and $D w_{M O R}$, RMSEP was 0.15 and $0.13, r_{p}^{2}$ was 0.79 and 0.91 , RPD was 2.0 and 3.2, and the bias was 0.032 and 0.036 , respectively. The relationships between measured and NIR-predicted values for both indicators and their associated prediction residuals are shown in Fig. 12.

The current results support previous research (Tsuchikawa and Schwanninger 2013) that reported a statistical link between NIRS spectra and mechanical properties 
Fig. 11 Mean spectrum and standard deviation of the differential spectra $S_{\text {diff }}$. The red ribbons (lengthwise stripes) show the excluded wavenumbers for PLSR. a $D w_{M O E}$ prediction, range 1: 3900-4104 $\mathrm{cm}^{-1}$, range 2: $4211-4420$ $\mathrm{cm}^{-1}$, range 3: 4632-4929 $\mathrm{cm}^{-1}$, range 4: $5079-5145 \mathrm{~cm}^{-1}$, range 5: $6163-7243 \mathrm{~cm}^{-1}$, range 6: $8080-8539 \mathrm{~cm}^{-1}$. b $D w_{M O R}$ prediction. range 1: 3900-4107 $\mathrm{cm}^{-1}$, range 2: $4200-4423 \mathrm{~cm}^{-1}$, range 3: 4624-4933 $\mathrm{cm}^{-1}$, range 4: $5068-5145 \mathrm{~cm}^{-1}$, range 5: $6144-7343 \mathrm{~cm}^{-1}$, range 6 : $8079-8604 \mathrm{~cm}^{-1}$
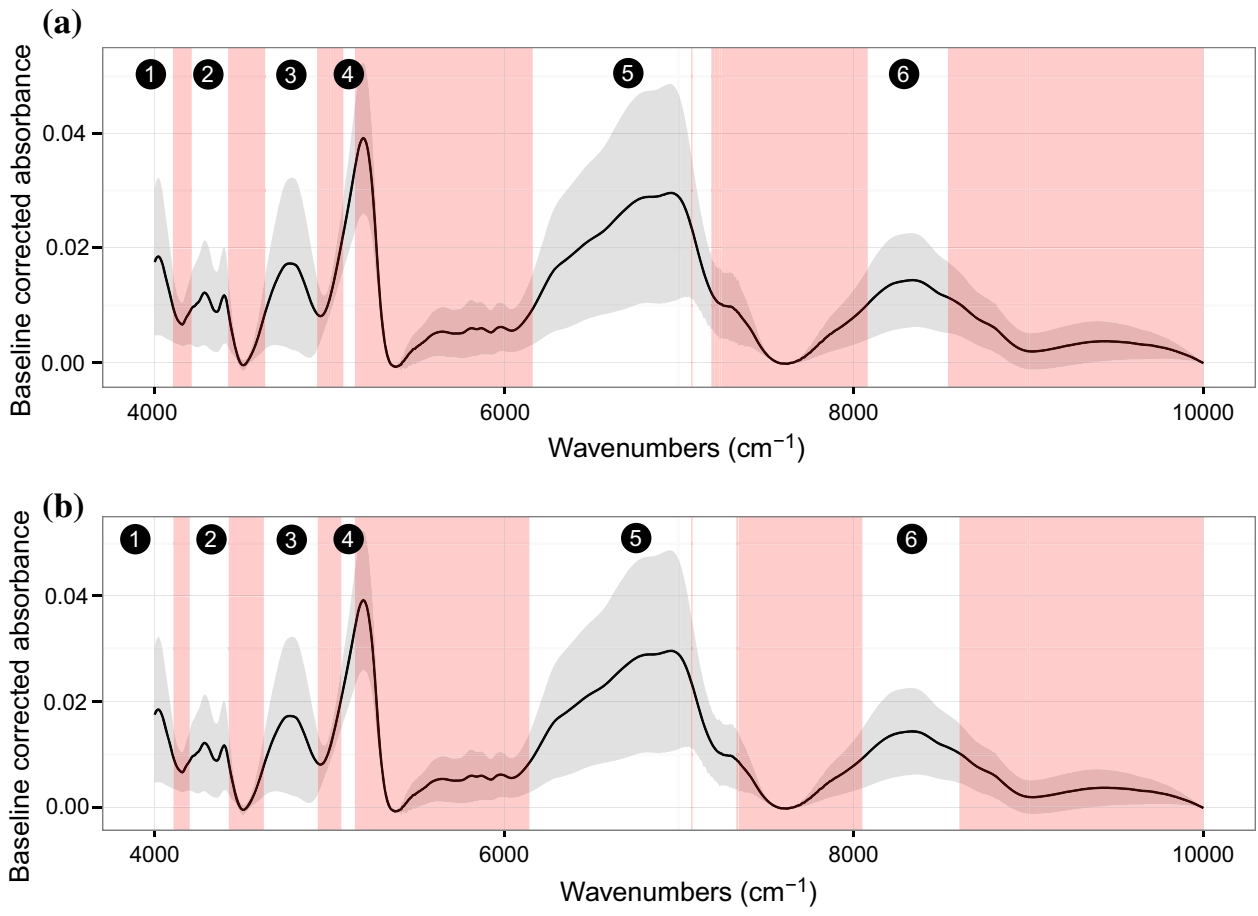

Table 2 Main model characteristics developed for predicting indicators $D w_{M O E}$ and $D w_{M O R}$

\begin{tabular}{|c|c|c|c|c|c|c|c|c|c|c|}
\hline \multirow{3}{*}{$\begin{array}{l}\text { Mechanical } \\
\text { indicator }\end{array}$} & \multicolumn{6}{|c|}{ Calibration phase, $\mathrm{n}=109$} & \multicolumn{4}{|c|}{ Validation phase, $\mathrm{n}=47$} \\
\hline & \multicolumn{3}{|c|}{ Calibration } & \multicolumn{3}{|c|}{ LOO cross-validation } & \multicolumn{4}{|c|}{ Prediction } \\
\hline & $\mathrm{NF}$ & $r_{c}^{2}$ & RMSEC & $\mathrm{NF}$ & $r_{v}^{2}$ & RMSECV & $r_{p}^{2}$ & RMSEP & RPD & Bias \\
\hline$D w_{M O E}$ & 11 & 0.75 & 0.14 & 11 & 0.64 & 0.16 & 0.79 & 0.15 & 2.0 & 0.032 \\
\hline$D w_{M O R}$ & 11 & 0.85 & 0.13 & 11 & 0.77 & 0.16 & 0.91 & 0.13 & 3.2 & 0.036 \\
\hline
\end{tabular}

$N F$ number of factors, $R M S E C$ root mean squared error of calibration, $R M S E C$ root mean squared error of cross validation, $R M S E P$ root mean squared error of prediction, $R P D$ relative percent difference ratio of $\mathrm{SD}$ to RMSEP, Bias the difference of the mean of the predicted versus the mean of the true values using PLSR. However, while most studies work with intact wood, the current models establish relationships between NIRS differential spectra $S_{\text {diff }}$ and losses in MOE and MOR of decayed wood (Table 2). Green et al. (2010a) already used NIRS and PLSR to predict the level of decay in wood measured by compression strength in particular. They obtained appreciable results with $r_{p}^{2}$ of 0.69 and RPD of 2.61 (model with raw spectra). While this study showed that NIRS could predict wood decay, it used Gloeophyllum trabeum (brown rot type) to inoculate samples. The current study shows similar results $\left(r_{p}^{2} 0.79\right.$ and RPD 2.0 for $D w_{M O E}$ prediction. $r_{p}^{2} 0.91$ and RPD 3.2 for $D w_{M O R}$ prediction) for predicting the level of decay in silver fir wooden strips inoculated with a microbial community.

Finally, from a practical standpoint, these methods may be applied to real structures by means of core collection (Noetzli et al. 2008). Spectral acquisition of cores could be directly acquired at different parts of the cores or on milled cores (Schimleck 2008). Cores $5 \mathrm{~mm}$ in diameter have very limited mechanical damage against the diameter of the logs generally used in ecological engineering structures (diameter above $150 \mathrm{~mm}$ ) and are easy to reduce into powder. This would give practitioners quantitative information about the level of decay of parts located by their expertise. However, the method needs to be tested previously on beams similar to those used in ecological engineering structures, i.e. wooden logs. The mean intact spectrum of silver fir should also be enriched by spectra from other locations to ensure that it covers the heterogeneity of the species.

\section{Conclusion}

The main purpose of this study was to predict degradation indicators of silver fir wooden strips decayed by a microbial community using NIRS. Two indicators of the level 
Fig. 12 Comparison between measured and predicted values for $D w_{M O E}$ (a) and $D w_{M O R}(\mathbf{c})$. The empty dots indicate the calibration set and the filled dots indicate the validation set. Prediction residuals $D w_{M O E}(\mathbf{b})$ and $D w_{M O R}(\mathbf{d})$ of for the validation set
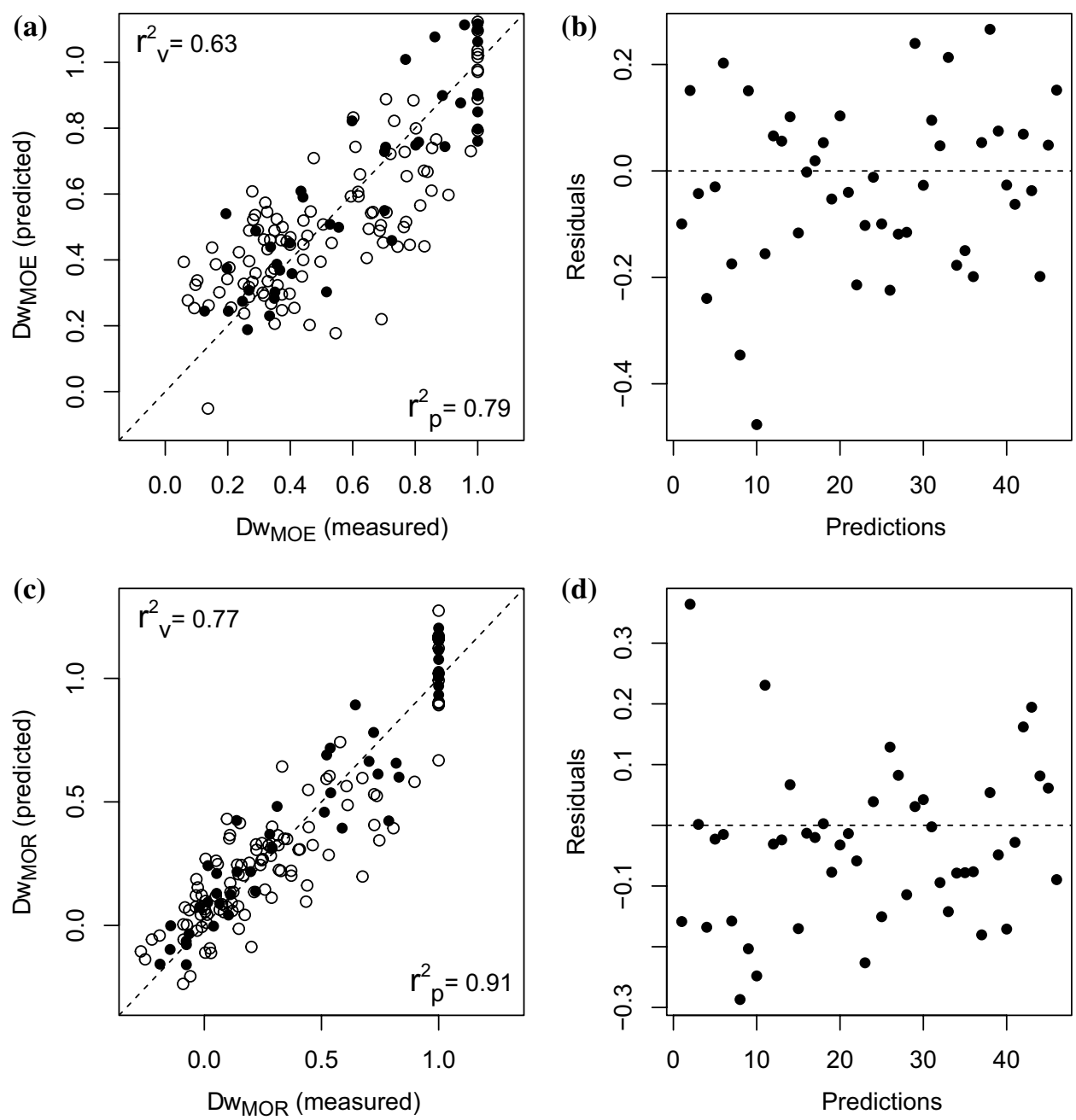

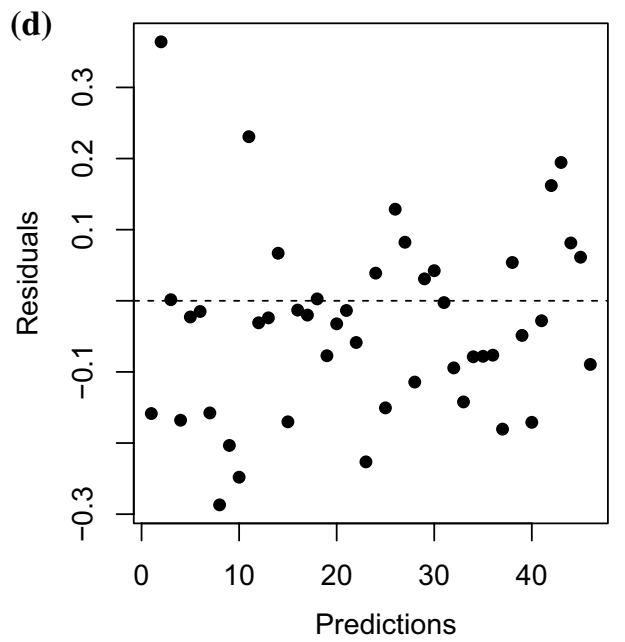

of decay, $D w_{M O E}$ and $D w_{M O R}$, were developed allowing an accurate evaluation of the level of decay in terms of mechanical properties. These indicators were related to the near infra-red spectra seen as estimators of the chemical compounds. The analysis of the NIRS spectra of intact and decayed milled samples confirmed the NIRS sensitivity to decay even under semi-controlled conditions. This sensitivity is observable over the full spectral range and nonattributable to one specific chemical compound (i.e. lignin, cellulose or hemicellulose). Based on these observations, two models were developed from spectra to predict $D w_{M O E}$ and $D w_{M O R}$ using PLSR. Predicted and measured values were highly correlated with $r_{p}^{2}$ values of $0.79\left(D w_{M O E}\right)$ and $0.91\left(D w_{M O R}\right)$. These models can provide useful tools for the rapid screening of wood residual properties of structural members. For that purpose, complementary investigations are needed. The scattering effects may be corrected by several methods, the multiplicative scatter corrections in particular (Rinnan et al. 2009), that should be compared in the context of the assessment of the level of decay. Furthermore, the method has been intentionally developed with a data set of limited variability (samples were sampled from one $\log$ ). The generalisation of the method with a data set taking into account an extensive variability is necessary.

Acknowledgements This work has been financed within the framework of projects SEDALP (Interreg Alpine space program. 20132015) and Arc Environnement (Rhône Alpes Region, France).

\section{References}

Beleites C, Sergo V (2012) hyperSpec: a package to handle hyperspectral data sets in $\mathrm{R}$

Bellon-Maurel V, Fernandez-Ahumada E, Palagos B, Roger JM, McBratney A (2010) Critical review of chemometric indicators commonly used for assessing the quality of the prediction of soil attributes by NIR spectroscopy. TrAC Trends Anal Chem 29(9):1073-1081

Blanchette RA (1995) Degradation of the lignocellulose complex in wood. Can J Bot 73(S1):999-1010

Cécillon L, Cassagne N, Czarnes S, Gros R, Brun JJ (2008) Variable selection in near infrared spectra for the biological characterization of soil and earthworm casts. Soil Biol Biochem 40(7):1975-1979 
CEN (2005) EN 1995-1-1. Eurocode 5-design of timber structures-Part 1-1-General rules: general rules and rules for buildings. Tech. rep., Brussels, Belgium

CEN (2014) EN 252:2013. Field test method for determining the relative protective effectiveness of a wood preservative in ground contact. Tech. rep., CEN, Brussels, Belgium

CIRAD (2011) Tropix 7 version 7.5.1. doi:10.18167/74726f706978

Curling S, Clausen C, Winandy J (2002) Relationships between mechanical properties, weight loss, and chemical composition of wood during incipient brown-rot decay. For Prod $\mathbf{J}$ 52(7-8):34-39

Dackermann U, Crews K, Kasal B, Li J, Riggio M, Rinn F, Tannert T (2013) In situ assessment of structural timber using stress-wave measurements. Mater Struct 47(5):787-803

Dinwoodie JM (2000a) Deformation under load. In: Timber: its nature and behaviour, 2nd edn. CRC Press, p 274

Dinwoodie JM (2000b) Strength and failure in timber. In: Timber: its nature and behaviour, 2nd edn. CRC Press, p 274

Fackler K, Schwanninger M (2012) How spectroscopy and microspectroscopy of degraded wood contribute to understand fungal wood decay. Appl Microbiol Biotechnol 96(3):587-599

Fackler K, Schmutzer M, Manoch L, Schwanninger M, Hinterstoisser B, Ters T, Messner K, Gradinger C (2007a) Evaluation of the selectivity of white rot isolates using near infrared spectroscopic techniques. Enzyme Microb Technol 41(67):881-887

Fackler K, Schwanninger M, Gradinger C, Hinterstoisser B, Messner K (2007b) Qualitative and quantitative changes of beech wood degraded by wood-rotting basidiomycetes monitored by Fourier transform infrared spectroscopic methods and multivariate data analysis. FEMS Microbiol Lett 271(2):162-169

Fackler K, Schwanninger M, Gradinger C, Srebotnik E, Hinterstoisser B, Messner K (2007c) Fungal decay of spruce and beech wood assessed by near-infrared spectroscopy in combination with uniand multivariate data analysis. Holzforschung 61(6):680-687

Gonzalez-Rodrigo B, Esteban L, de Palacios P, Garca-Fernndez F, Guindeo A (2013) Variation throughout the tree stem in the physical-mechanical properties of the wood of Abies alba Mill. From the Spanish Pyrenees. Madera Bosques 19(2):87-107

Green B, Jones PD, Nicholas DD, Schimleck LR, Shmulsky R (2010a) Non-destructive assessment of Pinus spp. wafers subjected to Gloeophyllum trabeum in soil block decay tests by diffuse reflectance near infrared spectroscopy. Wood Sci Technol 45(3):583-595

Green B, Jones PD, Schimleck LR, Nicholas DD, Shmulsky R (2010b) Rapid assessment of southern pine decayed by G. trabeum by near infrared spectra collected from the radial surface. Wood Fiber Sci 42(4):450-459

Green B, Jones PD, Nicholas DD, Schimleck LR, Shmulsky R, Dahlen J (2012) Assessment of the early signs of decay of Populus deltoides wafers exposed to Trametes versicolor by near infrared spectroscopy. Holzforschung 66(4):515-520

Haines D, Leban JM, Herb C (1996) Determination of Young's modulus for spruce, fir and isotropic materials by the resonance flexure method with comparisons to static flexure and other dynamic methods. Wood Sci Technol 30(4):253-263

Ishizuka S, Sakai Y, Tanaka-Oda A (2012) Quantifying lignin and holocellulose content in coniferous decayed wood using nearinfrared reflectance spectroscopy. J For Res 19(1):233-237

Kasal B, Tannert T (eds) (2011) In situ assessment of structural timber, RILEM state of the art reports, vol 7. Springer, Dordrecht

Kelley SS, Jellison J, Goodell B (2002) Use of NIR and pyrolysisMBMS coupled with multivariate analysis for detecting the chemical changes associated with brown-rot biodegradation of spruce wood. FEMS Microbiol Lett 209(1):107-111
Kelley SS, Rials TG, Groom LR, So CL (2004) Use of near infrared spectroscopy to predict the mechanical properties of six softwoods. Holzforschung 58(3):252-260

Leinonen A, Harju AM, Venlinen M, Saranp P, Laakso T (2008) FTNIR spectroscopy in predicting the decay resistance related characteristics of solid Scots pine (Pinus sylvestris L.) heartwood. Holzforschung 62(3):284-288

Liland KH, Mevik BH (2014) Baseline: baseline correction of spectra

Mevik BH, Cederkvist HR (2004) Mean squared error of prediction (MSEP) estimates for principal component regression (PCR) and partial least squares regression (PLSR). J Chemom 18(9):422-429

Mevik BH, Wehrens R, Liland KH (2013) pls: partial least squares and principal component regression

Mkip R, Linkosalo T (2011) A non-destructive field method for measuring wood density of decaying logs. Silva Fennica. doi:10.14214/sf.91

Nadler B, Coifman RR (2005) The prediction error in CLS and PLS: the importance of feature selection prior to multivariate calibration. J Chemom 19(2):107-118

Noetzli K, Boell A, Graf F, Sieber T, Holdenrieder O (2008) Influence of decay fungi, construction characteristics, and environmental conditions on the quality of wooden check-dams. For Prod J 58(4):72-79

Ottoson E (2013) Succession of wood-inhabiting fungal communities. PhD thesis, Swedish University of Agricultural Sciences, Uppsala

Previati M, Canone D, Bevilacqua I, Boetto G, Pognant D, Ferraris S (2012) Evaluation of wood degradation for timber check dams using time domain reflectometry water content measurements. Ecol Eng 44:259-268

Przewloka SR, Crawford DM, Rammer DR, Buckner DL, Woodward BM, Li G, Nicholas DD (2008) Assessment of biodeterioration for the screening of new wood preservatives: calculation of stiffness loss in rapid decay testing. Holzforschung 62(3):270-276

R Core Team (2013) R: a language and environment for statistical computing

Rinnan $\AA$, van den Berg F, Engelsen SB (2009) Review of the most common pre-processing techniques for near-infrared spectra. TrAC Trends Anal Chem 28(10):1201-1222

Rowell R (2012) Chemistry of wood strength. In: Handbook of wood chemistry and wood composites. CRC Press, p 703

Sandak A, Ferrari S, Sandak J, Allegretti O, Terziev N, Riggio M (2013) Monitoring of wood decay by near infrared spectroscopy. Adv Mater Res 778:802-809

Sandak A, Sandak J, Riggio M (2015a) Assessment of wood structural members degradation by means of infrared spectroscopy: an overview. Struct Control Health Monit. doi:10.1002/stc.1777

Sandak J, Sandak A, Riggio M (2015) Multivariate analysis of multisensor data for assessment of timber structures: principles and applications. Constr Build Mater 101(Part 2):1172-1180

Schimleck L (2008) Near-infrared spectroscopy: a rapid non-destructive method for measuring wood properties and its application to tree breeding. N Z J For Sci 38(1):14-35

Schimleck LR, Mora C, Daniels RF (2003) Estimation of the physical wood properties of green Pinus taeda radial samples by near infrared spectroscopy. Can J For Res 33(12):2297-2305

Schwanninger M, Rodrigues JC, Fackler K (2011) A review of band assignments in near infrared spectra of wood and wood components. J Near Infrared Spectrosc 19(5):287-308

Schwarze FWMR (2007) Wood decay under the microscope. Fungal Biol Rev 21(4): 133-170

Sila A, Terhoeven-Urselmans T (2013) soil.spec: soil spectral file conversion, data exploration and regression 
Stirling R, Trung T, Breuil C, Bicho P (2007) Predicting wood decay and density using NIR spectroscopy. Wood Fiber Sci 39(3):414-423

Stokland JN, Siitonen J, Jonsson BG (2012) Biodiversity in dead wood. Ecology, biodiversity, and conservation. Cambridge University Press, New York

Tsuchikawa S, Schwanninger M (2013) A review of recent near-infrared research for wood and paper (Part 2). Appl Spectrosc Rev 48(7):560-587
Wilcox W (1978) Review of literature on the effects of early stages of decay on wood strength. Wood Fiber Sci 9(4):252-257

Wold S, Sjstrm M, Eriksson L (2001) PLS-regression: a basic tool of chemometrics. Chemom Intell Lab Syst 58(2):109-130 